\title{
Inflammation in the vascular wall as an early event in atherosclerosis
}

\author{
S.M . Prescott, G . A . Z immerman, T. M. M cl ntyre, D. M . Stafforini
}

Eccles Institute of Human Genetics and Cardiovascular Research Training Institute, University of Utah, Salt Lake City, Utah, USA

The ability to attract leukocytes to the appropriate location in the vasculature is an essential homeostatic response. For example, it is crucial in defending against bacterial infection and in wound repair. However, the same processes, if they occur at the wrong location or under the inappropriate circumstances, can support pathological events. Many human diseases, particularly those with a strong inflammatory component, are the result of inappropriate recruitment and activation of leukocytes. Examples of this include the ischaemia-reperfusion syndromes in a variety of organs, the acute respiratory distress syndrome, and atherosclerosis.

We have examined the mechanisms by which leukocytes are recruited to the vascular wall in a spatially discrete manner and how their activation is regulated. Our initial studies focussed on polymorphonuclear leukocytes (PMNs) and we described a multi-step process by which they are recruited. The signal for recruitment begins with activation of endothelial cells in the appropriate location. We discovered that a variety of agonists including acute mediators such as thrombin, peptidoleukotrienes, histamine, and bradykinin can all stimulate endothelial cells to result in a surface that is now adhesive for expression of Pselectin on the surface of endothelial cells. This protein is stored in specialized secretory granules and is rapidly transferred to the surface of the cell, and serves to tether the leukocytes at the endothelial surface but does not itself activate the target leukocytes. The activation phase comes through the synthesis of a phospholipid mediator, platelet-activating factor (PAF), by the endothelial cells. This mediator is not

Corresponding author: S. M. Prescott, MD, Eccles Institute of Human Genetics, Building 533, Room 4220, University of Utah, Salt City, Utah 84112, USA

A bbreviations: PMN, Polymorphonuclear leukocytes; PAF, platelet-activating factor. secreted into the fluid phase but also is expressed the surface. From there it can bind to the receptor for PAF on the surface of the leukocyte. Binding to this receptor initiates an activation process that includes the up-regulation of the leukocytes' own adhesion proteins, the $\beta-2$ integrins, and other cellular responses such as a change in morphology, chemotaxis, emigration from the vasculature, and secretion of granular contents such as proteolytic enzymes and oxygen radicals. This process has the virtue of first attracting the leukocytes to the vascular wall surface and then only after they have attached at the proper location causing activation. The multi-step process provides an editing function so that cells that might adhere inappropriately but are not activated can release back into circulation and not damage the vasculature downstream.

In more recent, related studies, we have examined the recruitment of monocytes and macrophages under the same conditions. We have found that very similar responses occur. For example, P-selectin is an effective tether for these cells, which are associated with chronic inflammation. The recruitment and activation of monocytes is particularly important since it is these cells that form the characteristic signature of the atheroma - the foam cell. Once monocytes are recruited to a site destined to become an atherosclerotic plaque the macrophages take up LDL that has been modified and are converted into a lipid-laden cell. Moreover, these cells can synthesize and secrete cytokines and chemokines, which amplify the inflammatory reaction. We found that monocytes and macrophages that were adherent to P-selectin, but not other adhesion proteins, were primed for subsequent synthesis and secretion of cytokines and chemokines. However, this required a second molecule to be added - one that resulted in a signalling pathway. PAF could serve this role but other molecules such as a variety of chemokines also were 
effective. The remarkable synergy between binding to this adhesion protein and subsequent activation through a receptor-mediated process may be very important for the early phases of chronic inflammation in the vasculature, such as that which occurs at atherosclerotic sites.

Another way in which these usually physiological processes can be disrupted is by inappropriate activation of the endothelial cells. One such stimulus is oxidant damage. We discovered that exposure of endothelial cells to strongly oxidizing conditions resulted in the prolonged and enhanced expression of P-selectin on their surface which in vivo would serve to attract an excessive number of PMNs. In addition, the cells also secreted a compound with very similar properties to PAF. In a series of experiments we discovered that this compound was not authentic PAF but was a group of oxidized phospholipids that derived from free radical-mediated damage of membrane phospholipids. These compounds act through the PAF receptor, but exert pathological consequences since their synthesis is not controlled.

In both the physiological and pathological situations, a key issue is how the signal for inflammation is turned off. We have studied an enzyme in human plasma that catalyses the degradation of PAF and related oxidized phospholipids. This enzyme, PAF acetylhydrolase, is tightly associated with LDL and HDL. It is able to circulate in a fully active form since it is highly specific for these unusual phospholipids that is, it will not work on the usual phospholipids that comprise cell membranes and lipoprotein particles. We have purified this enzyme and cloned a cDNA. Recombinant PAF acetylhydrolase is effective in preventing inflammation in a variety of animal models and may be effective in acute, severe inflammation in humans. Whether it can apply a sufficient off-signal to prevent atherosclerosis is an interesting question that is under examination.

In summary, the recruitment of leukocytes, both PMNs and monocytes, to the vascular wall is a multistep process involving an adhesion protein and a separate activating stimulus from the endothelial cells, and a complex response by the leukocyte. Under physiological circumstances this is a tightly regulated process that results in the recruitment of cells to an appropriate site in the vasculature with activation only after emigration to the tissue. Under pathological circumstances, the tightly regulated process is disrupted and cells are recruited in excessive number or at inappropriate spaces. 